Original article

\title{
A multicentre qualitative study assessing implementation of an Enhanced Recovery After Surgery program ${ }^{\text {th }}$
}

\author{
D. Martin a, D. Roulin a , F. Grass a, V. Addor a , O. Ljungqvist ${ }^{\text {b }}$, N. Demartines ${ }^{\text {a, *, }}$, \\ M. Hübner ${ }^{\mathrm{a}}$ \\ a Department of Visceral Surgery, Lausanne University Hospital CHUV, Lausanne, Switzerland \\ ${ }^{\mathrm{b}}$ Faculty of Medicine and Health, School of Health and Medical Sciences, Department of Surgery, Örebro University, Örebro, Sweden
}

\section{A R T I C L E I N F O}

\section{Article history:}

Received 12 July 2017

Accepted 25 October 2017

\section{Keywords:}

Enhanced recovery after surgery

Implementation

Qualitative study

\begin{abstract}
S U M M A R Y
Background \& aims: The existence of enhanced recovery specific guidelines (ERAS) is not enough to change patient management practice since many barriers exist to successful ERAS implementation. The present survey aimed to analyse motivations for implementation as well as encountered difficulties and challenges. Further, relevance and importance of perioperative care items and postoperative recovery targets were assessed.

Methods: A multicentre qualitative study was conducted between August and December 2016 among surgeons, anaesthesiologists and nurses from implemented ERAS centres in Switzerland $(\mathrm{n}=16)$ and Sweden ( $\mathrm{n}=14)$. An online survey (31 closed questions) was sent by email, with reminders at 4, 8 and 12 weeks.

Results: Seventy-seven out of 146 experts completed the survey (response rate 52.7\%). Main motivations to implement ERAS were the expectation to reduce complications (91\%), higher patient satisfaction (73\%) and shorter hospital stay (62\%). The application of ERAS program represented major changes in clinical practice for $57 \%$ of participants without significant differences between various specialities (surgeons: $63 \%$, nurses: $63 \%$, anaesthesiologists: $36 \%, \mathrm{p}=0.185)$. The most important barriers for straightforward implementation were time restraints (69\%), opposing colleagues (68\%) and logistical reasons (66\%). The 3 most frequently cited patient-related barriers to adopt ERAS were opposing personality (52\%), comorbidities (49\%) and language barriers (31\%).

Conclusions: Implementing ERAS care into practice was challenging and required important changes in clinical practice for all involved specialities. Main reasons for implementation were the expectation to reduce complications and hospital stay with improved patients' satisfaction. Main barriers were time restraints, reluctance to change and logistics.
\end{abstract}

๑ 2017 Elsevier Ltd and European Society for Clinical Nutrition and Metabolism. All rights reserved.

\section{Introduction}

Enhanced Recovery After Surgery (ERAS) pathways reduce overall morbidity, length of hospital stay and costs by incorporating multimodal optimization of perioperative care in colorectal, upper gastrointestinal, liver and pancreatic surgery, and more recently in urology, gynaecology and orthopaedic surgery [1-11].

\footnotetext{
4resented to the $5^{\text {th }}$ ERAS Society World Congress, May 2017, Lyon, France; published in abstract form as Clinical Nutrition ESPEN (Volume 19, June 2017, Pages 81). Presented as a poster to the $104^{\text {th }}$ Annual Congress of the Swiss Society of Surgery, June 2017, Bern, Switzerland; published in abstract form as Swiss Knife 2017; 14: special edition.

* Corresponding author. Department of Visceral Surgery, Lausanne University Hospital CHUV, 1011 Lausanne, Switzerland. Fax: +41 213142411.

E-mail address: demartines@chuv.ch (N. Demartines).
}

Previous research assessed adherence to the protocol and improvement of clinical outcomes to evaluate completeness and success of ERAS implementation [12-14]. In daily clinical practice, it may be complex to introduce and apply simultaneously a relatively high number of new interventions among many providers and across hospital services [15]. Qualitative studies allow to define those barriers and provide multidimensional additional information [16]. Implementation challenges have been attributed to a variety of key areas, namely related to patient, staff, practice and resources [17]. Therefore, evidence-based principles of ERAS are not widely adopted and implementation is lagging way behind the evidence [18].

The present survey aimed to analyse the reasons and motivations that made centres decide to implement ERAS as well as 
difficulties encountered and challenges during implementation. Secondly, the relevance and importance of postoperative recovery targets and perioperative care items were assessed as well.

\section{Material and methods}

\subsection{Study design and participants}

A multicentre qualitative study was conducted among ERAS teams from centres in Switzerland $(n=16)$ and Sweden $(n=14)$ that had implemented ERAS principles by participating in the ERAS Society Implementation Program (www.erassociety.org [2,19]). Each centre had an ERAS coordinator on site. At least one member of the surgical, nursing and anaesthetic team, considered as trained in ERAS, was asked to participate in each institution. This survey concerned all different surgical specialities. The questions included participant demographics as well as difficulties and challenges during ERAS implementation. The relevance and importance of pre-defined postoperative recovery targets and perioperative care items were assessed by asking participants to rate each component on a semantic differential scale (not important at all: 0, very important: 10). ERAS perceived protocol deviation was also assessed with a similar scale (never: 0 , every time:10). This evaluation was compared between the different care providers. The survey was sent by email using online cloud-based software (Survey Monkey ${ }^{\circledR}$, Palo Alto, CA, United States). Automatic reminders were sent at 4,8 and 12 week intervals. The survey included 31 closed questions and took an estimated $10 \mathrm{~min}$ for completion. Data were collected between August and December 2016.

\subsection{Statistical analyses}

Descriptive statistics for categorical variables were reported as number and percentage, while continuous variables were reported as median and interquartile range (IQR) or means and standard deviation (SD) as appropriate. Pearson's chi square test was used for comparison of categorical variables. Continuous variables were compared with the Kruskal-Wallis test or Mann-Whitney U test when appropriate. A p value $\leq 0.05$ was considered statistically significant. All statistical analyses were two-sided and performed using SPSS 22.0 software (SPSS Inc., Chicago, IL).

\subsection{Ethics}

This survey targeted medical staff only, and did not affect patients in any way. The local Committee for Medical and Health Research Ethics suggested that no formal ethical approval was required for this survey.

\section{Results}

\subsection{Demographics}

Seventy-seven out of 146 experts completed the survey yielding a response rate of $52.7 \%$. Fifty-eight responses were from Swiss participants (response rate 63\%) and 19 from Swedish participants (response rate 37\%). Demographics of responding experts are summarized in Table 1 . Response rate was not significantly different between specialities (surgeons 65\%, anaesthesiologists 65\% and nurses $57 \%, \mathrm{p}=0.790$ ).

\subsection{Reasons for ERAS implementation}

The main reasons for initiating the ERAS implementation were the following expected benefits: reducing complications (91\%),
Table 1

Demographics of the survey participants.

\begin{tabular}{llll}
\hline & Swiss, $\mathrm{n}=58$ & Swedish, $\mathrm{n}=19$ & Overall, $\mathrm{n}=77$ \\
\hline Mean age (SD) & $44.1(8.3)$ & $46.1(9.2)$ & $44.7(8.5)$ \\
Male gender, n (\%) & $31(53)$ & $9(47)$ & $40(52)$ \\
Speciality, n (\%) & & & \\
$\quad$ Surgeons & $25(43)$ & $8(42)$ & $33(43)$ \\
$\quad$ Anaesthesiologists & $13(22)$ & $4(21)$ & $17(22)$ \\
$\quad$ Nurses & $20(35)$ & $7(37)$ & $27(35)$ \\
Mean years working & $3.0(1.6)^{\mathrm{a}}$ & $4.9(2.8)^{\mathrm{a}}$ & $3.5(2.1)$ \\
$\quad$ with ERAS (SD) & & & \\
\hline
\end{tabular}

Abbreviation: SD, standard deviation.

${ }^{\text {a }}$ Indicates statistical significance, $\mathrm{p}$ value $\leq 0.05$, Mann-Whitney U test.

higher patient satisfaction (73\%), and shorter hospital stay (62\%). Further, in descending order, the participants aimed for improved patients' quality of life (32\%), reduced costs $(26 \%)$ or workload $(9 \%)$ and for publicity and marketing (6\%).

\subsection{Implementation challenges}

The application of ERAS program represented major changes in clinical practice for $57 \%$ of the responding experts. A total of $63 \%$ of surgeons, $63 \%$ of nurses and $35 \%$ of anaesthesiologists $(p=0.185)$ stated that ERAS induced major changes in their practice. Preoperative ERAS-specific education required an additional 15-30 min for $49 \%$ of the responders, more than $30 \mathrm{~min}$ (42\%) and less than 15 min (9\%) and was delivered by nurses (60\%), surgeons (10\%), or both together (30\%). Participants were asked to evaluate the perceived rate of intentional ERAS protocol deviation according to specialities, which is described in Fig. 1. Thirty-six participants (47\%) claimed to conduct ERAS meetings once a month, $18 \%$ twice a month, $15 \%$ once a week and the remaining $20 \%$ less than once per month. In terms of importance for success of the implementation, patient's preoperative education was rated at 10/10 (IQR 8-10), the presence of an ERAS coordinator at 10/10 (IQR 8-10) and staff education at 9/10 (IQR 8-10). Forty-six participants (60\%) felt that is was more difficult to change habits to ERAS for colleagues with training in more traditional care pathways compared to those with recent training.

\subsection{Barriers to implementation}

The most important barriers to ERAS implementation were time restraints (69\%), opposition from colleagues (68\%) and logistical reasons (composing/distributing memos and care maps, continuous training, 66\%). Less important barriers were opposition from the administration, money restraints and patientrelated factors (53\%, 34\% and $10 \%$, respectively). The 3 most frequently cited patient-related barriers to implementation where opposing personality (52\%), co-morbidities (49\%) and language barriers (31\%). The 3 most relevant co-morbidities impeding straightforward application of ERAS were mental illness (64\%), cardiovascular disease (38\%) and disability (27\%). Barriers related to age and socio-cultural level were cited by $23 \%$ and $22 \%$ of participants, respectively. Forty percent of participants stated that ERAS could equally be applied regardless of age. In contrast, $42 \%$ of participants considered patients over 80 years of age to be unsuitable ERAS candidates. Early discharge was judged to be difficult by 22 experts (29\%) due to insufficient resources (home care and rehabilitation centres). Conversely, in terms of importance for successful ERAS protocol compliance, patient personality was rated at 7/10 (IQR 6-8), co-morbidities at 7/10 (IQR 5-7) and age at 4/10 (IQR 2-7). 


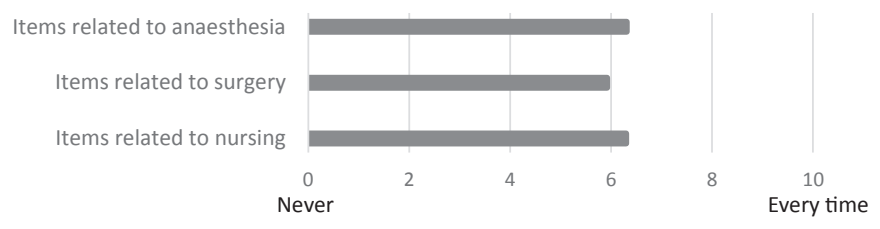

Fig. 1. ERAS perceived protocol deviation according to speciality.

\subsection{ERAS postoperative recovery targets and perioperative care items}

The relevance and importance of perioperative care items and postoperative recovery targets according to ERAS experts are described in Figs. 2 and 3. Most experts felt that ERAS implementation reduced length of stay by 3 days (26\%), 2 days (25\%), more than 4 days (13\%) and 1 day (9\%). Fourteen participants did not know (18\%) and 4 reported no decrease in length of stay (5\%). Seventy-three participants (95\%) believed that length of stay might still decrease following ERAS implementation.

\section{Discussion}

This survey among professionals involved in and leading ERAS pathways in their local hospital is one of the first analysing the motivation and difficulties of ERAS implementation in daily clinical practice. Some interesting discrepancies but also similarities of view between subjective feeling of ERAS care providers have been highlighted. In fact, implementation required important changes of clinical practice in order to achieve improved outcomes, and the amount of work and energy should not be underestimated. It starts

\section{Preoperative items}

Avoiding oral bowel

preparation

Avoiding anaesthetic

sedative medication

Fasting kept at absolute minimum

Oral carbohydrate loading

Counseling

\section{Intraoperative items}

Using epidural analgesia when indicated

Avoiding drain

Avoiding nasogastric tube

Antimicrobial prophylaxis and skin preparation

Optimising fluid balance

\section{Postoperative items}

Stimulating gut motility

Allowing normal diet at will after the operation

Promoting early and scheduled mobilisation

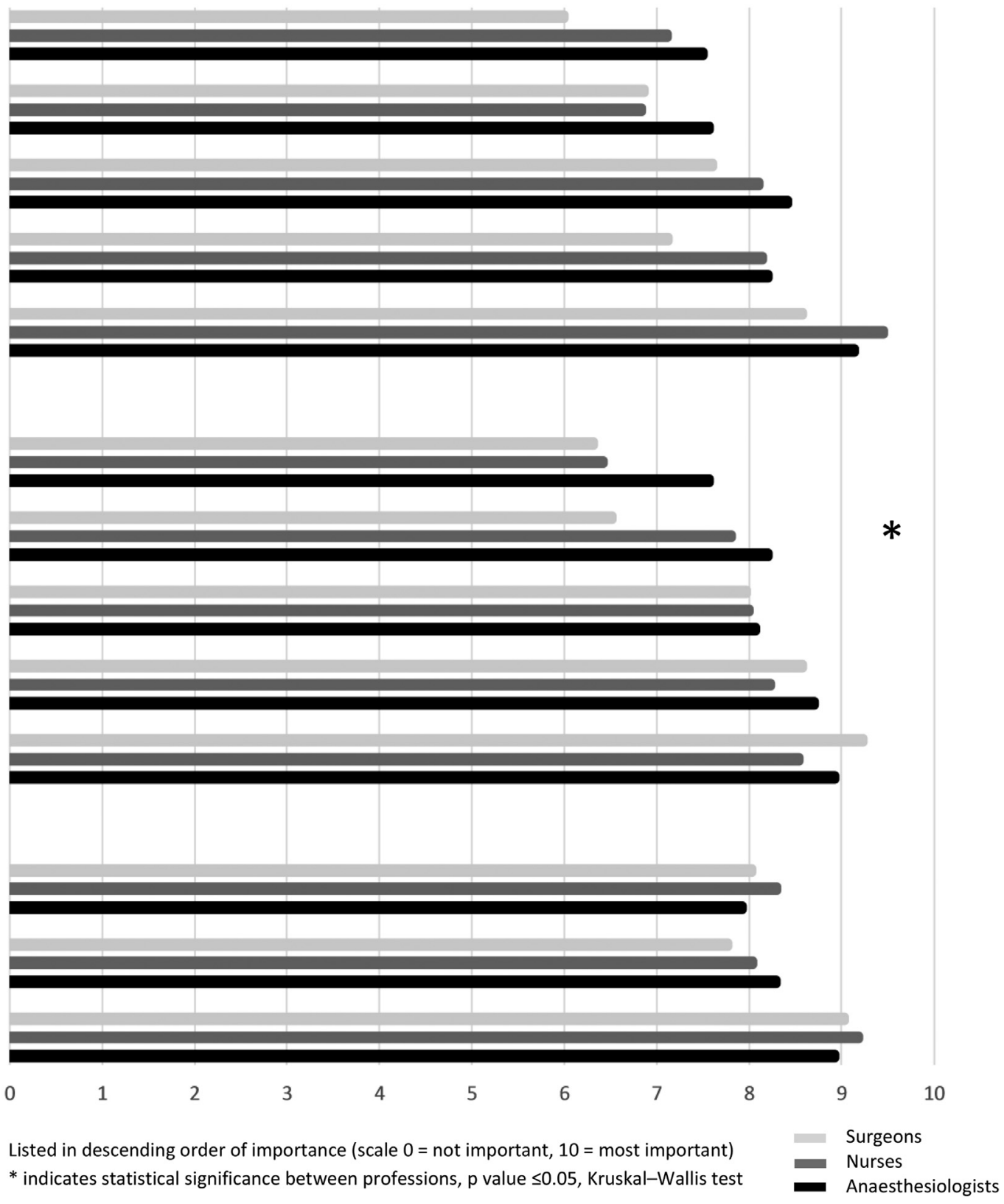

Fig. 2. Comparison of ERAS experts' rating of perioperative care items. 
To be discharged from hospital as soon as possible?

To have bowel movement as soon as possible?

To be completely free of pain upon movement?

To be able to return to all daily activities as soon as possible?

To be able to eat and drink as soon as possible?

To be independently mobile in hospital as soon as possible?

To be completely free of pain at rest?

To be completely free of nausea?

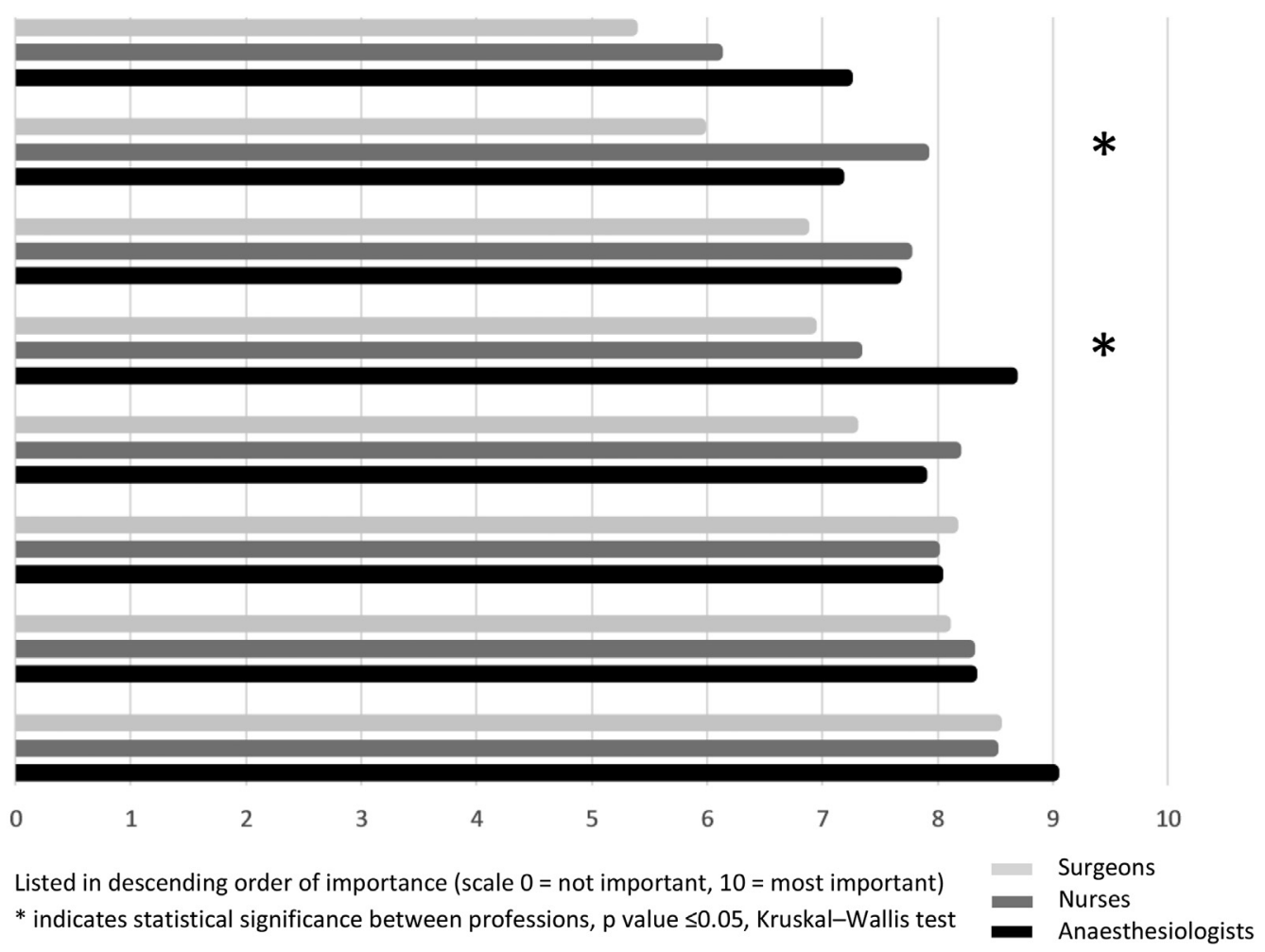

Fig. 3. Comparison of ERAS experts' rating of recovery targets. with main barriers, namely time restraints, reluctance to change and logistics. Main factors in favour of ERAS implementation were the expectations to reduce complications and hospital stay as well as to improve patients' satisfaction.

Participants to this survey, considered as trained in ERAS, were well balanced in terms of demographics of the typical ERAS teams comprising of surgeons, anaesthesiologists and nurses. However, the Swedish group had a significantly longer experience with the ERAS program. Age and experience of professionals may have influenced attitude toward ERAS implementation, as described by a majority of participants (60\%). Most centres conducted meetings once a month, which is less frequent than recommended by the ERAS Society and which risks to be insufficient [14,20]. At the outset during the actually implementation program, a unit should meet weekly to audit compliance and implement necessary changes. After some time, and with increased experience of the ERAS group, the frequency of the team meetings can be reduced, but attendance at meetings must remain an established commitment for every team member [20]. In this study, participants reported that the majority of patients received preoperative nursing education, which lasted usually between 15 and $30 \mathrm{~min}$. Pearsall et al. conducted an exploratory study using interviews, and identified lack of education for patients and their family as a common problematic [21]. As a consequence, in our implemented hospitals, a patient education booklet and daily activity log were developed and handed to patients when they consented for surgery.

Surprisingly, intentional ERAS protocol deviation was perceived to be considerable $(>50 \%)$ in the present study. In a consecutive cohort of patients undergoing elective colorectal surgery, the decision to deviate from the ERAS clinical pathway was mainly a medical decision [22]. However, reasons for non-compliance were medically justified in $78 \%$ of the study participants, which still leaves room for improvement in almost a quarter of deviations. Given the difficulty in determining the relative importance of each individual item, the idea of a flexible and individualised approach rather than a rigid protocol has also been postulated [23-25]. However, it has repeatedly been shown that when variation of care is reduced and evidence based ERAS protocols are being instituted, outcomes improve. A minimal overall compliance of $70 \%$ was recently shown to be necessary to achieve improved clinical outcomes [26]. Thus, deviation from protocols should be done for a strong medical reason only. Another crucial point for successful implementation is the identification of an ideally enthusiastic team leader. As demonstrated in the present survey, the median importance of an ERAS coordinator was 10 out of 10 on a semantic differential scale. The ERAS coordinator, often a specialised nurse, fills a key role within ERAS team, with time devoted to spread information and managing practical matters [20].

Despite overwhelming evidences supporting ERAS, adoption and implementation of ERAS pathways remain scarce across Europe $[23,27,28]$. Several reasons might account for this low interest. A better understanding of potential barriers and drawbacks is one important factor $[23,28,29]$. In the present study, most important barriers to implementation were lack of time, opposing colleagues and logistical reasons. Two studies independently reported general resistance to change, time and staff restraints, as well as poor communication, collaboration and coordination between departments as main reasons for failure [21,30]. In an American retrospective study, 26\% of barriers and enablers to ERAS implementation occurred at the site and unit levels, with a provider focus $26 \%$ of the time, a patient focus $26 \%$ of the time, and a system focus $22 \%$ of the time [19]. Concerning staff, lack of interest was reported, particularly among nurses and anaesthesiologists [31]. One possible explanation is that unlike surgeons, these latter do not follow patients from the beginning to the end of perioperative management, and do not necessarily see the actual benefits of the ERAS program. Large numbers of different caregivers including anaesthesiologists might impede straightforward implementation [15]. As demonstrated in this study, the application of ERAS program represented major changes in clinical practice for $63 \%$ of 
surgeons, $63 \%$ of nurses and $35 \%$ of anaesthesiologists. Although these results are not statistically different, probably because of the small sample size, anaesthesiologists seem less concerned with the change in their practice generated by ERAS implementation. A recent comprehensive consensus review for anaesthesia practice in patients undergoing gastrointestinal surgery within an ERAS program aimed promulgating anaesthesiologists' involvement as mandatory in implementation process [32]. In the current literature, lack of education of the multidisciplinary team was also identified as drawback. To increase multi-institutional collaboration, regular conference calls to share best practice among sites and exchange about experiences and difficulties have been reported to be useful [21]. In a observational study, compliance to ERAS items was found to be low and affecting recovery and length of hospital stay due to different disciplines providing care for the same patients [23]. Implementation of protocol requires a dedicated ERAS team consisting of surgeons, anaesthesiologists and nursing staff, and needs a continuous education to sustain adherence [14,33,34].

In the present study and according to ERAS experts, barriers related to the patient were their opposing personality. However, a so-called opposing personality is rather the results of lack of understanding of the benefit of ERAS pathway. This may be corrected by improved information and discussion with the patient and his relative to empower the patient as supporting active actor for its entire perioperative process. This capital information requires time and patience as well as adequate communication skills. In addition, language difficulties and age above 80 years may increase the difficulty of understanding the process. For these reasons, individually adapted preadmission counselling is thus crucial to prepare patients to assume his active role [35]. Contrary as supposed by $42 \%$ of experts in the present study, elderly patients are well suited for ERAS pathway: a recently published retrospective study showed that adherence to the ERAS pathway was equally high in patients aged over 70 years [36]. Despite more co-morbidities, older patients did not experience more complications and recovery was similar. ERAS did therefore not require specific adaptations for elderly. Likewise, ERAS can be applied to emergent cases and appears to work also over the weekend even with reduced staff $[37,38]$. It has to be emphasized that inclusion of patient perspectives is critical to identify challenges and facilitators to implement ERAS [39].

The relative importance of ERAS postoperative recovery targets and perioperative care items was previously reported $[40,41]$. Nausea and pain free recovery and the ability to eat, drink and move quickly after surgery are of utmost importance. Concerning perioperative care, the items "preoperative counselling", "optimizing fluid balance", and "promoting early and scheduled mobilisation" received the highest scores in a survey conducted during the first international ERAS congress 2012 as well as in the present survey [40]. The reproducibility of these results indicates uniformity in the perception of recovery targets over time by ERAS experts and trainees [40]. Functional recovery, as food tolerance without nausea, adequate pain control and ability to mobilize, is consistently considered the most important target for recovery and might be used when defining the recovered patient in future research and audits, as concluded also by other surveys amongst international experts [41,42].

Several limitations of the present study need to be addressed. ERAS guidelines for colorectal surgery have been published before other specialities [43]. Most centres have therefore implemented ERAS for colorectal surgery first, then gradually the other specialities. This is especially valid for referral centres, but less for smaller peripheral centres, where ERAS is mainly applied to colorectal surgery, which represented most centres included in this study. This present survey concerned all different specialities, and might explain why many barriers to implementation have been identified. Furthermore, not all members of the multidisciplinary perioperative ERAS team were interviewed. In particular, dieticians, physiotherapists, and especially residents who are important pillars for proper functioning of the perioperative team were not included. Moreover, patients themselves were not interviewed but their insights should be part of another study. The focus of the present study was on ERAS leaders which were supposed to be familiar with the pathway. The importance and role of each specialist in the implementation of ERAS was not evaluated. The response rate was surprisingly low for a survey among health care professionals who should be a priori particularly motivated for enhanced recovery, but provided the large amount of online surveys regularly sent around the web on various subject, responders are today more difficult to motivate, thus the response rate may be regarded as acceptable compared to other survey and is still representative [44]. Another potential explanation might be lack of time and especially lack of interest from some care providers, to which ERAS implementation could have been imposed. Finally, audit is an integral part of the ERAS Implementation Program and the only way to know whether there is improvement. The audit was performed at least every other week as part of the implementation program. However, the specific question regarding the value of audit was not assessed in this present survey.

In conclusion, implementing evidence-based ERAS care into practice was perceived as challenging and required important commitment and changes in clinical practice for all involved specialities. Main motivation to implement ERAS pathway was the expectation to reduce complications and hospital stay, as well as to improve patients' satisfaction. Functional recovery, as tolerance of food without nausea, adequate pain control and ability to mobilize, was consistently considered the most important target for recovery among involved specialities.

\section{Conflict of interest}

OL founded and owns stock in Encare AB, registered in Sweden that manages the ERAS Interactive Audit System. No other conflict of interest.

\section{Funding source}

None.

\section{Statement of authorship}

All the authors contributed to the work and approved the final version of the manuscript. Particularly, contributions were: study design: DM, MH, OL, ND; Data collection: DM, DR, FG, VA; Data analyses and interpretation: DM, DR, FG, VA, OL, ND, MH; Manuscript draughting: DM, DR, FG, MH; Critical revision of the manuscript: DM, DR, FG, VA, OL, ND, MH.

\section{Acknowledgements}

The authors wish to thank the participants who answered the survey anonymously in the different ERAS centres in Switzerland and Sweden.

\section{References}

[1] Greco M, Capretti G, Beretta L, Gemma M, Pecorelli N, Braga M. Enhanced recovery program in colorectal surgery: a meta-analysis of randomized controlled trials. World J Surg 2014;38(6):1531-41. 
[2] Roulin D, Donadini A, Gander S, Griesser AC, Blanc C, Hubner M, et al. Costeffectiveness of the implementation of an enhanced recovery protocol for colorectal surgery. Br J Surg 2013;100(8):1108-14.

[3] Bond-Smith G, Belgaumkar AP, Davidson BR, Gurusamy KS. Enhanced recovery protocols for major upper gastrointestinal, liver and pancreatic surgery. Cochrane Database Syst Rev 2016;2, Cd011382.

[4] Cerantola Y, Valerio M, Persson B, Jichlinski P, Ljungqvist O, Hubner M, et al. Guidelines for perioperative care after radical cystectomy for bladder cancer: enhanced Recovery After Surgery (ERAS((R))) society recommendations. Clin Nutr 2013;32(6):879-87.

[5] Azhar RA, Bochner B, Catto J, Goh AC, Kelly J, Patel HD, et al. Enhanced recovery after urological surgery: a contemporary systematic review of outcomes, key elements, and research needs. Eur Urol 2016;70(1):176-87.

[6] Melloul E, Hubner M, Scott M, Snowden C, Prentis J, Dejong CH, et al. Guidelines for perioperative care for liver surgery: enhanced recovery After surgery (ERAS) society recommendations. World J Surg 2016;40(10): 2425-40.

[7] Nelson G, Altman AD, Nick A, Meyer LA, Ramirez PT, Achtari C, et al. Guidelines for pre- and intra-operative care in gynecologic/oncology surgery: enhanced Recovery After Surgery (ERAS(R)) Society recommendations-Part I. Gynecol Oncol 2016;140(2):313-22.

[8] Nelson G, Altman AD, Nick A, Meyer LA, Ramirez PT, Achtari C, et al. Guidelines for postoperative care in gynecologic/oncology surgery: Enhanced Recovery After Surgery $(\operatorname{ERAS}(\mathrm{R}))$ Society recommendations-Part II. Gynecol Oncol 2016;140(2):323-32.

[9] Scott NB, McDonald D, Campbell J, Smith RD, Carey AK, Johnston IG, et al. The use of Enhanced Recovery After Surgery (ERAS) principles in Scottish orthopaedic units-an implementation and follow-up at 1 year, 2010-2011: a report from the Musculoskeletal Audit, Scotland. Arch Orthop Trauma Surg 2013;133(1):117-24

[10] Thanh NX, Chuck AW, Wasylak T, Lawrence J, Faris P, Ljungqvist O, et al. An economic evaluation of the Enhanced Recovery after Surgery (ERAS) multisite implementation program for colorectal surgery in Alberta. Can J Surg J Can de Chir 2016:59(6):415-21.

[11] Stone AB, Grant MC, Pio Roda C, Hobson D, Pawlik T, Wu CL, et al. Implementation costs of an enhanced recovery after surgery program in the United States: a financial model and sensitivity analysis based on experiences at a quaternary academic medical center. J Am Coll Surg 2016;222(3):219-25.

[12] Gillissen F, Hoff C, Maessen JM, Winkens B, Teeuwen JH, von Meyenfeldt MF, et al. Structured synchronous implementation of an enhanced recovery program in elective colonic surgery in 33 hospitals in The Netherlands. World Surg 2013;37(5):1082-93.

[13] Miller TE, Thacker JK, White WD, Mantyh C, Migaly J, Jin J, et al. Reduced length of hospital stay in colorectal surgery after implementation of an enhanced recovery protocol. Anesth Analg 2014;118(5):1052-61.

[14] Martin D, Roulin D, Addor V, Blanc C, Demartines N, Hubner M. Enhanced recovery implementation in colorectal surgery-temporary or persistent improvement? Langenbecks Arch Surg 2016;401(8):1163-9.

[15] Gotlib Conn L, McKenzie M, Pearsall EA, McLeod RS. Successful implementation of an enhanced recovery after surgery programme for elective colorectal surgery: a process evaluation of champions' experiences. Implement Sci 2015;10:99.

[16] Strauss A, Corbin J. Basics of qualitative research: techniques and procedures for developing grounded theory. 2nd ed. Thousand Oaks, CA: Sage Publications; 1998.

[17] Lyon A, Solomon MJ, Harrison JD. A qualitative study assessing the barriers to implementation of enhanced recovery after surgery. World J Surg 2014;38(6): 1374-80.

[18] Kehlet H, Wilmore DW. Evidence-based surgical care and the evolution of fast-track surgery. Ann Surg 2008;248(2):189-98.

[19] Gramlich LM, Sheppard CE, Wasylak T, Gilmour LE, Ljungqvist O, BasualdoHammond C, et al. Implementation of Enhanced Recovery After Surgery: a strategy to transform surgical care across a health system. Implement Sci 2017;12(1):67.

[20] Ljungqvist O, Scott M, Fearon KC. Enhanced recovery after surgery: a review. JAMA Surg 2017:152(3):292-8.

[21] Pearsall EA, Meghji Z, Pitzul KB, Aarts MA, McKenzie M, McLeod RS, et al. A qualitative study to understand the barriers and enablers in implementing an enhanced recovery after surgery program. Ann Surg 2015:261(1):92-6.

[22] Roulin D, Muradbegovic M, Addor V, Blanc C, Demartines N, Hubner M. Enhanced recovery after elective colorectal surgery - reasons for noncompliance with the protocol. Dig Surg 2017;34(3):220-6.
[23] Maessen J, Dejong CH, Hausel J, Nygren J, Lassen K, Andersen J, et al. A protocol is not enough to implement an enhanced recovery programme for colorectal resection. Br J Surg 2007;94(2):224-31.

[24] Nicholson A, Lowe MC, Parker J, Lewis SR, Alderson P, Smith AF. Systematic review and meta-analysis of enhanced recovery programmes in surgical patients. Br J Surg 2014;101(3):172-88.

[25] Lyon A, Payne CJ, Mackay GJ. Enhanced recovery programme in colorectal surgery: does one size fit all? World J Gastroenterol 2012;18(40):5661-3.

[26] Jurt J, Slieker J, Frauche P, Addor V, Sola J, Demartines N, et al. Enhanced recovery after surgery: can we rely on the key factors or do we need the bel ensemble? World J Surg 2017;41:2464.

[27] Donohoe CL, Nguyen M, Cook J, Murray SG, Chen N, Zaki F, et al. Fast-track protocols in colorectal surgery. Surg J R Coll Surg Edinb Irel 2011:9(2): 95-103.

[28] Gustafsson UO, Hausel J, Thorell A, Ljungqvist O, Soop M, Nygren J. Adherence to the enhanced recovery after surgery protocol and outcomes after colorectal cancer surgery. Arch Surg 2011;146(5):571-7.

[29] The impact of enhanced recovery protocol compliance on elective colorectal cancer resection: results from an international registry. Ann Surg 2015;261(6):1153-9.

[30] Ament SM, Gillissen F, Moser A, Maessen JM, Dirksen CD, von Meyenfeldt MF, et al. Identification of promising strategies to sustain improvements in hospital practice: a qualitative case study. BMC Health Serv Res 2014;14:641.

[31] Kahokehr A, Robertson P, Sammour T, Soop M, Hill AG. Perioperative care: a survey of New Zealand and Australian colorectal surgeons. Colorectal Dis 2011;13(11):1308-13.

[32] Feldheiser A, Aziz O, Baldini G, Cox BP, Fearon KC, Feldman LS, et al. Enhanced Recovery After Surgery (ERAS) for gastrointestinal surgery, part 2: consensus statement for anaesthesia practice. Acta Anaesthesiol Scand 2016;60(3): 289-334.

[33] Polle SW, Wind J, Fuhring JW, Hofland J, Gouma DJ, Bemelman WA. Implementation of a fast-track perioperative care program: what are the difficulties? Dig Surg 2007;24(6):441-9.

[34] Gillissen F, Ament SM, Maessen JM, Dejong CH, Dirksen CD, van der Weijden T, et al. Sustainability of an enhanced recovery after surgery program (ERAS) in colonic surgery. World J Surg 2015;39(2):526-33.

[35] Galli E, Fagnani C, Laurora I, Marchese C, Capretti G, Pecorelli N, et al Enhanced Recovery After Surgery (ERAS) multimodal programme as experienced by pancreatic surgery patients: findings from an Italian qualitative study. Int J Surg 2015;23(Pt A):152-9.

[36] Slieker J, Frauche P, Jurt J, Addor V, Blanc C, Demartines N, et al. Enhanced recovery ERAS for elderly: a safe and beneficial pathway in colorectal surgery. Int J Colorectal Dis 2017;32(2):215-21.

[37] Romain B, Grass F, Addor V, Demartines N, Hubner M. Impact of weekday surgery on application of enhanced recovery pathway: a retrospective cohort study. BMJ Open 2016;6(10), e011067.

[38] Roulin D, Blanc C, Muradbegovic M, Hahnloser D, Demartines N, Hubner M. Enhanced recovery pathway for urgent colectomy. World J Surg 2014;38(8): 2153-9.

[39] Alawadi ZM, Leal I, Phatak UR, Flores-Gonzalez JR, Holihan JL, Karanjawala BE, et al. Facilitators and barriers of implementing enhanced recovery in colorectal surgery at a safety net hospital: a provider and patient perspective. Surgery 2016;159(3):700-12.

[40] Aahlin EK, von Meyenfeldt M, Dejong CH, Ljungqvist O, Fearon KC, Lobo DN, et al. Functional recovery is considered the most important target: a survey of dedicated professionals. Perioper Med (Lond) 2014;3:5.

[41] Hughes M, Coolsen MM, Aahlin EK, Harrison EM, McNally SJ, Dejong CH, et al. Attitudes of patients and care providers to enhanced recovery after surgery programs after major abdominal surgery. J Surg Res 2015;193(1):102-10.

[42] Fiore Jr JF, Bialocerkowski A, Browning L, Faragher IG, Denehy L. Criteria to determine readiness for hospital discharge following colorectal surgery: an international consensus using the Delphi technique. Dis Colon Rectum 2012;55(4):416-23.

[43] Gustafsson UO, Scott MJ, Schwenk W, Demartines N, Roulin D, Francis N, et al. Guidelines for perioperative care in elective colonic surgery: Enhanced Recovery After Surgery (ERAS(R)) Society recommendations. Clin Nutr 2012;31(6):783-800.

[44] Demartines N, Freiermuth O, Mutter D, Heberer M, Harder F. Knowledge and acceptance of telemedicine in surgery: a survey. J Telemed Telecare 2000;6(3):125-31. 